\title{
Sequential Decoding of Lattice Codes
}

\author{
Vahid Tarokh ${ }^{1} \quad$ Alexander Vardy ${ }^{2} \quad$ Kenneth Zeger ${ }^{3}$ \\ ${ }^{1}$ AT\&T Laboratories, 600 Mountain Ave., Murray Hill, NJ 07974, USA (tarokh@research.att.com) \\ ${ }^{2}$ Coordinated Science Laboratory, University of Illinois, 1308 W. Main Street, \\ Urbana, IL 61801, USA (vardy@golay.csl.uiuc.edu) \\ ${ }^{3}$ Department of Electrical and Computer Engineering, University of California at San Diego, \\ La Jolla, CA 92093-0407, USA (zeger@ucsd.edu)
}

\begin{abstract}
We consider lattice tree-codes based on a lattice $\Lambda$ having a finite trellis diagram $T$. Such codes are easy to encode and benefit from the structure of $\Lambda$. Sequential decoding of lattice tree-codes is studied, and the corresponding Fano metric is derived. An upper bound on the running time of the sequential decoding algorithm is established, and found to resemble the Pareto distribution. Our bound indicates that the order of label groups in $T$ plays an important role in determining the complexity of sequential decoding. Furthermore, it is proved that lattice tree-codes of arbitrarily high rate, based on $\Lambda$ and $T$, can be sequentially decoded with the same complexity, and without any possibility of buffer overflow.
\end{abstract}

\section{INTRODUCTION}

Although Viterbi decoding of lattice codes is optimal, its computational complexity grows exponentially as a function of the coding gain [5]. This result motivates the development of sub-optimal trellis decoding algorithms for lattice codes that remain efficient for large trellises.

One such algorithm is sequential decoding. It is arguably the most well-known sub-optimal trellis decoding algorithm. Historically, it has been studied for decoding convolutional codes, and has an older history than maximum likelihood decoding [3]. The success of sequential decoding for convolutional codes, both in theoretical and practical terms, and the growing applications of lattice codes in communications motivate a similar study for lattice codes. Such a study is the subject of this work.

We demonstrate that sequential decoding is a computationally efficient near-optimal technique for decoding lattice codes. Furthermore, in contrast to convolutional codes, there is no possibility of buffer overflow in sequential decoding of lattices.

\section{Fano Metric for Lattice Codes}

In 1963, Fano [1] heuristically postulated a metric for sequential decoding of convolutional codes. Massey [4] later proved that this metric is, in fact, the correct criterion for this purpose. This metric is now known as the Fano metric.

Herein, we use the argument of Massey [4] to derive the optimal Fano metric for sequential decoding of Euclidean codes. We then introduce lattice tree-codes. These codes are easy to encode and are suitable for sequential decoding purposes. Moreover, they benefit complexity-wise from the structure of

\footnotetext{
* This work was supported in part by the National Science Foundation and by the JSEP grant N00014-9610129.
}

the defining lattice. We derive an appropriate sequential decoding metric for these codes.

\section{Running Time Analysis}

We analyze the running time of the sequential decoding algorithm for lattice tree-codes. An analog of Pareto's distribution is obtained, which upper bounds the expected running time of the proposed algorithm.

We demonstrate that the label group complexity plays an important role in the sequential decoding of lattice codes. Since the label group complexity is known to grow at most linearly in terms of the coding gain, sequential decoding offers an attractive alternative to Viterbi decoding of lattice codes. This is somewhat analogous to the case of convolutional codes. It is well known that Viterbi decoding of convolutional codes with large constraint length has exponential complexity. Nevertheless, sequential decoding has been successfully implemented in practice.

\section{Performance Analysis and Discussion}

It is known that the performance of a sequential decoder at high SNR is essentially identical to that of maximum likelihood decoding. This is proved for convolutional codes in [2] and can be easily generalized to lattice codes. Simulation results also indicate that this is indeed the case when sequential decoding is applied to the trellis diagram of lattices.

We point out that the family of Barnes-Wall lattices, whose trellises can be constructed from tensor products, are attractive for sequential decoding purposes. The higher-dimensional members of this family, however, are not attractive for trellisbased maximum-likelihood decoding, as their asymptotic trellis complexity grows without bound [5].

An interesting difference between sequential decoding of lattices and that of convolutional codes is that there is no possibility of buffer overflow in the former case. This is due to the finiteness of the trellis in this case, and could make lattice tree-codes attractive for some applications.

\section{REFERENCES}

[1] R.M.Fano, "A heuristic discussion of probabilistic decoding," IEEE Trans. Inform. Theory, vol. 9, pp. 64-73, 1963.

[2] G.D. Forney, Jr., "Convolutional codes II: maximum likelihood decoding," Inform. and Control, vol. 25, pp. 222-266, 1974.

[3] G.D. Forney, Jr., "Convolutional codes III: sequential decoding," Inform. and Control, vol. 25, pp. 267-297, 1974.

[4] J.L. Massey, "Variable-length codes and the Fano metric," IEEE Trans. Inform. Theory, vol. 18, pp. 196-198, 1972.

[5] V. Tarokh, Trellis Complexity versus the Coding Gain of Lattice-based Communication Systems, Ph.D. Thesis, 1995. 